\title{
COMPOSTOS BIOATIVOS (COMPOSTOS FENÓLICOS E ATIVIDADE ANTIOXIDANTE) E CARACTERÍSTICAS FÍSICO-QUÍMICAS DA CASCA DO FRUTO DO MANDACARU (cereus jamacaru)
}

\section{BIOATIVE COMPOUNDS (PHENOLIC COMPOUNDS AND ANTIOXIDANT ACTIVITY) AND PHYSICO-CHEMICAL CHARACTERISTICS OF MANDACARU FRUIT SHELL (cereus jamacaru)}

\author{
Isabela Alves dos Santos ${ }^{1}$; Tamires dos Santos Pereira²; Raphael Lucas Jacinto Almeida ${ }^{3}$ \\ Cecília Elisa de Sousa Muniz ${ }^{4}$ José Nilton Silva ${ }^{5}$
}

DOI: $\underline{\text { https://doi.org/10.31692/978-65-991061-7-0.250-257 }}$

\section{RESUMO}

O mandacaru (Cereus jamacaru) é uma planta nativa do Brasil, presente em grande quantidade no Nordeste do país. Seus frutos são fontes de fitonutrientes importantes para a saúde, justificando assim o seu consumo, além de servir como estímulo ao seu melhor aproveitamento, apresentando um alto conteúdo de compostos fenólicos totais e uma elevada atividade antioxidante. Após o consumo de frutas, muitas vezes as cascas dos alimentos são desperdiçadas. O reaproveitamento de alimentos é uma solução para diversos problemas atuais simples de ser tomada, como desperdício, geração de resíduo, poluição, entre outros, basta uma pequena mudança nos hábitos. Com o intuito de estimular, cada vez mais, pesquisadores das mais diversas áreas em estudar a casca do fruto do mandacaru e explorá-la cientificamente em benefício da população que dela depende, demonstrando vantagens existentes no desenvolvimento de novas tecnologias para obter produtos a partir da casca deste fruto, é necessário, inicialmente, fazer uma caracteriação físico química, bem como avaliar a presença de compostos bioativos neste material. Desta forma, este trabalho teve por objetivo caracterizar a casca do fruto do mandacaru por meio das análises de teor de água, cinzas, $\mathrm{pH}$, acidez, brix, atividade de água, açúcares redutores, compostos fenólicos totais e atividade antioxidante (método do radical ABTS). Os frutos utilizados neste trabalho foram colhidos no município de Sertânia, Pernambuco. Para a casca deste fruto foram obtidos valores de $92,04 \%$ de umidade em base úmida, 1,3\% de cinzas, 1,53 de grau ${ }^{\circ}$ Brix, 0,7\% para a acidez, expressa em ácido cítrico, $\mathrm{pH}$ de 4,41, atividade de água de 0,984, açúcares redutores de $1,46 \%$ expresso em glicose. Para os compostos bioativos, apresentou valor de 326,78 (mg ácido gálico/ $100 \mathrm{~g}$ ) de compostos fenólicos totais e uma atividade antioxidante, pelo método do radical ABTS de 7,23 ( $\mu \mathrm{M}$ Trolox/g).

Palavras-Chave: Método ABTS, Caracterização, Compostos Fenólicos Totais, Atividade Antioxidante

\begin{abstract}
\footnotetext{
${ }^{1}$ Engenharia Química, UFCG, isabelaeq@gmail.com

${ }^{2}$ Engenharia de Processos, UFCG, tsantosp16@gmail.com

${ }^{3}$ Engenharia Química, UFCG, raphaelqindustrial@gmail.com

${ }^{4}$ Química, UFCG, ceciliamuniz.qi@gmail.com

${ }^{5}$ Doutor em Engenharia Química, UFCG, nilton@eq.ufcg.edu.br
}

Mandacaru (Cereus jamacaru) is a native plant of Brazil, which is present in a large quantity in the northeastern region of the same country. Their fruits are sources of phytonutrients which are important for health, thus justifying their consumption, besides serving as a stimulus to their best use, presenting a high content of total phenolic compounds and a high antioxidant activity. After consuming fruits, often the barks of food are wasted. The reuse of 
food is a solution to several current problems that are simple to take, such as waste, generation of waste, pollution, among others, a small change in habits is enough. With the purpose of stimulating, more and more, researchers of the most diverse areas to study the bark of the mandacaru fruit and to explore it scientifically for the benefit of the population that depends on it, demonstrating advantages in the development of new technologies to obtain products from the peel of this fruit, it is necessary, initially, to make a physical chemical characterization, as well as to evaluate the presence of bioactive compounds in this material. The objective of this work was to characterize the bark of the mandacaru fruit by means of the analysis of water content, ashes, $\mathrm{pH}$, acidity, brix, water activity, reducing sugars, total phenolic compounds and antioxidant activity ). The fruits used in this work were collected in the municipality of Sertânia, Pernambuco. For the bark of this fruit, values of $92.04 \%$ of moisture were obtained in wet basis, $1.3 \%$ of ashes, 1.53 degrees ${ }^{\circ}$ Brix, $0.7 \%$ for acidity, expressed as citric acid, $\mathrm{pH}$ of 4,41 , water activity of 0.984 , reducing sugars of $1.46 \%$ expressed as glucose. For the bioactive compounds, it presented a value of 326.78 (mg gallic acid / $100 \mathrm{~g}$ ) of total phenolic compounds and an antioxidant activity, by the ABTS radical method of 7.23 ( $\mu \mathrm{M}$ Trolox / g).

Keywords: ABTS Method, Characterization, Total Phenolic Compounds, Antioxidant Activity

\section{INTRODUÇÃO}

O Cereus jamacaru, conhecido popularmente como o mandacaru do nordeste brasileiro, é uma cactácea nativa do Brasil, com grande capacidade de captação e retenção de água, o que proporciona sua sobrevivência em longos períodos de estiagem (TAYLOR et al., 2004).

Ainda são poucos os estudos na literatura sobre os frutos desta planta, apesar de serem encontrados em grandes quantidades de fevereiro a setembro, no Nordeste brasileiro, não sendo tão explorado comercialmente. Desta forma, visando a importância de usufruir ao máximo a diversidade da fruticultura brasileira é necessário que a casca do fruto do mandacaru seja avaliada, nutricional e quimicamente.

Os compostos fenólicos têm se tornado cada vez mais evidentes devido aos efeitos benéficos à saúde por sua atividade antioxidante, e por serem responsáveis por possíveis ações farmacológicas. Segundo Melo et al., 2017 e Calado et al., 2016, analisando frutos do mandacaru do estado da Paríba, encontraram valores expressivos com relação aos compostos fenólicos e atividade antioxidante.

\section{FUNDAMENTAÇÃO TEÓRICA}

Os compostos fenólicos são compostos biologicamente ativos, capazes de interferir a nível molecular no organismo. É devido a este fator que nos últimos anos, uma atenção crescente tem sido dedicada a ação desses compostos, presentes em alimentos de origem vegetal na manutenção da saúde humana (MARTINS et al., 2016; SILVA et al., 2010; WINK, 
2016).)

Muito do sabor, odor e coloração de diversos vegetais que apreciamos são gerados por estes, podendo ser classificados em dois grupos: os flavonóides e não flavonóides (SILVA, 2014).

Pesquisas têm demonstrado que os compostos fenólicos apresentam grande interesse nutricional por contribuir com propriedades anticancerígenas, antioxidante, hipoglicêmico, efeitos cardioprotetores e vasodilatadores, hipolipidico e anti-inflamatório, já que estão relacionado com a capacidade antioxidativa. Variações de magnitude de compostos fenólicos têm sido conferidas a fatores como a forma de cultivo, tempo de colheita e tipo de solo (KALINOWSKA et al., 2014; ALU'DATT et al., 2017; BELMIRO et al., 2017; MAGALHÃES et al., 2017).

\section{METODOLOGIA}

Os experimentos foram realizados no Laboratório de Engenharia Bioquímica (LEB) da Unidade Acadêmica de Engenharia Química no Centro de Ciências e Tecnologia da Universidade Federal de Campina Grande - Paraíba, com exceção da análise de atividade de água, a qual foi conduzida no Laboratório de Termodinâmica de Engenharia Química do Centro de Tecnologia da Universidade Federal da Paraíba - Campus João Pessoa (DEQ/CT/UFPB).

A casca do fruto do mandacaru caracterizada neste trabalho foi obtido no município de Sertânia, Pernambuco. Posteriormente, estes frutos foram submetidos à seleção para retirada de frutos com lesões visíveis e infecções. A uniformidade de cor e tamanho também foram levados em conta.

Para os experimentos foi apenas utilizada a casca do fruto do mandacaru, por isso, após ser feita a seleção, esta foi separada da polpa, como pode ser visto na Figura 1.

Figura 1: Casca e polpa do fruto do mandacaru, respectivamente. Fonte: Própria
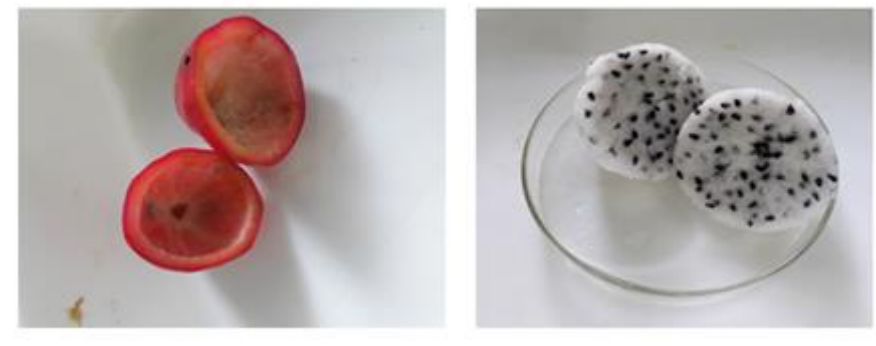

As análises realizadas foram: Teor de água, cinzas, pH, sólidos solúveis totais, açúcares redutores, atividade de água, acidez total titulável, compostos fenólicos e atividade antioxidante. Todas as análises foram realizadas em triplicata e os resultados foram expressos 
como média e desvio.

Para determinação do teor de água foi utilizado o método gravimétrico na estufa a $105^{\circ} \mathrm{C}$ por $24 \mathrm{~h}$ (IAL, 2008). . O teor de umidade (em base úmida) foi obtido pela diferença entre massa inicial e final

A determinação de cinzas foi realizada em uma mufla a temperatura de $700^{\circ} \mathrm{C}$ por um período de 5 horas. Esse procedimento foi necessário para que houvesse a destruição total da matéria orgânica presente na amostra (IAL, 2008).

Utilizando uma proporção de 1:10, foi preparada uma solução com $20 \mathrm{~mL}$ de água destilada e 2 gramas de amostra em um bécker. Após homogeneização a suspensão foi deixada em repouso por um período de 30 minutos. Esta solução foi filtrada em um papel filtro contendo algodão. Posteriormente mediu-se o $\mathrm{pH}$ da solução aquosa em potenciômetro digital, previamente calibrado com as soluções padrões.

Os sólidos solúveis totais, expressos em $\left({ }^{\circ}\right.$ Brix), foram determinados pelo método refratométrico, com refratômetro com escala em grau Brix, de acordo com as normas do manual do Instituto Adolfo Lutz (IAL, 2008).

A determinação de açúcares redutores foi feita conforme metodologia de Miller (1959). O método é baseado na redução do ácido 3,5 dinitrosalicílico (DNS) a 3-amino-5nitrosalicilico, simultaneamente com a oxidação do grupo aldeído do açúcar ao grupo carboxílico.

A atividade de água foi determinada através de leitura direta da amostra no medidor de água Novasina LabMaster -aw,e foi utilizada a temperatura de $25^{\circ} \mathrm{C}$ para as leituras.

Dterminou-se a acidez total titulável através do método acidimétrico do manual do Instituto Adolfo Lutz (IAL, 2008) em que as amostras foram tituladas com solução padronizada de $\mathrm{NaOH} 0,1 \mathrm{~N}$ (os resultados expressos em percentagem de ácido cítrico)

A metodologia para análise de compostos fenólicos totais foi feita pelo método de Folin \& Ciocalteu, adaptado da EMBRAPA preconizado por WATERHOUSE (2006).

A análise da atividade antioxidante pelo método do radical ABTS foi realizada, conforme metodologia descrita por RUFINO et al (2007), onde a atividade antioxidante foi medida através da captura do radical 2,2- azinobis(3-etilbenzotiazolina-6-ácido sulfônico) (ABTS).

\section{RESULTADOS E DISCUSSÃO}

Na Tabela 1, são mostrados os valores médios e os desvios padrões da caracterização físico-química e compostos fenólicos totais para a casca do fruto do mandacaru in natura. 
Tabela 1: Caracterização da casca do fruto do mandacaru in natura. Fonte: Própria

\begin{tabular}{|c|c|}
\hline Parâmetros analisados & Média \pm Desvio Padrão \\
\hline Teor de água (\%) & $92,04 \pm 0,9$ \\
\hline Cinzas $(\%)$ & $1,3 \pm 0,3$ \\
\hline${ }^{\circ}$ Brix & $1,53 \pm 0,04$ \\
\hline Acidez (\% Ácido Cítrico) & $0,7 \pm 0,03$ \\
\hline $\mathrm{pH}$ & $4,41 \pm 0,01$ \\
\hline Atividade de água & $0,984 \pm 0,001$ \\
\hline Açúcares Redutores (\% em glicose) & $1,46 \pm 0,05$ \\
\hline Fenólicos Totais (mg ácido gálico/ $100 \mathrm{~g})$ & $326,78 \pm 4,35$ \\
\hline $\begin{array}{c}\text { Atividade Antioxidante }(\mu \mathrm{M} \text { Trolox/g de } \\
\text { amostra) }\end{array}$ & $7,23 \pm 0,19$ \\
\hline
\end{tabular}

O teor de água encontrado indica elevada quantidade de água na casca do fruto, característica esta que não favorece a sua conservação em condições ambientais

O valor médio de cinzas, apresentado na Tabela 10, quando comparado com a literatura está acima do valor encontrado por Silva et al. (2012), que foi de 0,44\%, indicando assim que o material estudado possui um maior teor de minerais. As cinzas em alimentos são consideradas como parâmetro de qualidade, pois quanto maior o seu valor, também são maiores os teores dos minerais.

Os sólidos solúveis totais ( ${ }^{\circ}$ Brix) são usados como índice de maturação para alguns frutos e indicam a quantidade de substâncias que se encontram dissolvidas no suco, constituído, na sua maioria de açúcares (CHAVES et al., 2004).

Silva et al. (2012) obtiveram valores de acidez variando entre 0,05 e 0,33 (\% ácido cítrico) para polpa e casca do fruto do mandacaru, respectivamente. Melo (2008) e Oliveira et al. (2004) determinaram para a polpa valores de 0,224 e 0,211(\% ácido cítrico), respectivamente. Diante desses valores nota-se que a acidez encontrada neste trabalho para a casca do fruto do mandacaru foi superior aos estudos encontrados na literatura.

Observa-se que o valor encontrado para o $\mathrm{pH}$ da casca do fruto do mandacaru in natura baseado na classificação de BARUFFALDI e OLIVEIRA (1998) a casca do fruto do mandacaru é considerada como alimento ácido ( $\mathrm{pH}$ entre 3,7 e 4,5).

Com relação ao fruto do mandacaru, nota-se que o valor de açúcares redutores (AR) da casca do fruto no presente trabalho foi inferior aos valores encontrados para polpa. Silva e Alves (2009) verificaram uma porcentagem maior de açúcares redutores para a polpa do fruto, com 5,76\%. Oliveira et al. (2004) e Melo (2008) encontraram valores de 9,54 e 10,88\% para polpa, respectivamente.

A atividade de água, corresponde à relação existente entre a pressão parcial de vapor de água na superfície do alimento $(p)$ e a pressão parcial de vapor de água pura $\left(p_{0}\right)$ a uma 
dada temperatura. É um parâmetro que varia de 0 a 1 , onde os alimentos ricos em água, apresentam valores acima de 0,90, os quais, nessas condições, sofrem facilmente contaminação microbiológica e reações enzimáticas e oxidativas.

O valor encontrado para o teor de fenólicos totais expresso em mg de ácido gálico/100 $\mathrm{g}$ da casca do fruto do mandacaru foi de 326,78 EAG (mg ácido gálico/100 g de polpa). Vieira et al (2011) avaliaram e encontraram os seguintes teores de fenólicos totais, expressos nessa mesma unidade, para os frutos da acerola (Malpighia emarginata DC.), 835,25 EAG, bacuri (Platonia insignis Mart.), 10,35 EAG, cajá (Spondias mombin L.), 70,92 EAG, caju (Anacardium occidentale), 201,61 EAG, goiaba (Psidium guajava), 104,76 EAG e tamarindo (Tamarindus indica L.), 23,57 EAG.

Para atividade antioxidante pelo método ABTS o valor encontrado no presente trabalho foi de 7,04 ( $\mu \mathrm{M}$ Trolox/ g de amostra) e encontra-se de acordo com os dados encontrados por Melo et al. (2017), que avaliando frutos do mandacaru colhidos no município de Barra de Santa Rosa-PB para três diferentes estádios de maturação, encontrou os valores de atividade antioxidade para casca deste fruto de 6,88, 8,31 e 10,14 ( $\mu \mathrm{M}$ Trolox/g de amostra).

\section{CONCLUSÕES}

As cascas deste fruto apresentam um grande potencial para o consumo in natura. caracterização da casca do fruto do mandacaru in natura permitiu conhecer o perfil físicoquímico do material, com teores de Fenólicos Totais e atividade antioxidante significativos.

\section{REFERÊNCIAS}

ALU'DATT, M.; RABABAH, T.; ALHAMAD, M. N.; ALMAHASNEH, M.; ALMAJWAL, A.; GAMMOH, S.; EREIFEJ, K.; JOHARGY, A.; ALLI,I. Areview of phenolic compounds in oil-bearing plants: Distribution, identification and occurrence of phenolic compounds. Food Chemistry, nº 218, pg 99-106, 2017.

BARUFFALDI, R.; OLIVEIRA, M. N. Fatores que condicionam a estabilidade de alimentos. In: BARUFFALDI, R.; OLIVEIRA, M. N. Fundamentos de tecnologia de alimentos. São Paulo: Atheneu. v. 3, p. 13-25, 1998.

BELMIRO, T. M. C.; PEREIRA, C. F.; PAIM, A. P. S. Red wines from South America: Content of phenolic compounds and chemometric distinction by origin. Microc (2017), doi: 10.1016/j.microc. 2017.03.018. 2017.

CALADO, J. A.; COSTA, F. B.; PEREIRA, M. M; D.; NASCIMENTO, A. M.; FORMIGA, A. S.; SILVA, M. S. ESTUDO DAS CARACTERÍSTICAS FÍSICO-QUÍMICAS DO FRUTO DO MANDACARU (Cereus jamacaru P.DC.) CULTIVADO NO SERTÃO PERNAMBUCANO. p. 1216-1219, 2016. 
IAL. Instituto Adolfo Lutz (São Paulo). Métodos físico-químicos para análise de alimentos. $4^{\circ}$ edição, $1^{\circ}$ edição digital, 2008.

KALINOWSKA, M.; BIELAWSKA, A.; LEWANDOWSKA-SIWKIEWICZ, H.; PRIEBE, W.; LEWANDOWSKI, W. Apples: Content of phenolic compounds vs. variety, part of apple and cultivation model, extraction of phenolic compounds, biological properties. Plant Physiology and Biochemistry, no 84, pg. 169-188, 2014..

MAGAlHÃES,S. C. Q.; TAVEIRA, M.T.; CABRITA,A. R. J.; FONSECA,A. J. M.; VALENTÃO,P.; ANDRADE,P. B. European marketable grain legume seeds: Further insight into phenolic compounds profiles. Food Chemistry, nº 215, pg.177-184, 2017.

MARTINS, N.; PETROPOULOS, S.; FERREIRA, I. C. F. R. Chemical composition and bioactive compounds of garlic (Allium sativum $L$.) as affected by pre-and post- harvest conditions: A review. Food Chemistry. v. 211. P. 41-50, 2016.

MELO, K. S.; Secagem de polpa do fruto do mandacaru. Dissertação (Mestrado em Engenharia Agrícola) - Programa de Pós-Graduação em Engenharia Alimentos da Universidade Federal de Campina Grande, Campina Grande, p. 152, 2008.

MELO, R. S.; SILVA, S. M.; SOUSA, A. S. B.; LIMA, R. P.; DANTAS, A. L.; DANTAS, R. L.; FIGUEIREDO, V. M. Maturação e qualidade de frutos de mandacaru (Cereus jamacaru P.DC.) de diferentes bioclimas do estado da Paraíba. Revista Agropecuária Técnica. v. 38, n. 3, p. 160-168, 2017.

MILLER, G. L. Use of dinitrosalicylic acid reagent for determination of reducing sugar. Analitical Chemistry, v.31, pg. 426-428, 1959.

OLIVEIRA, F. M. N.; ALEXANDRE, H. V.; FIGUEIRÊDO, R. M. F.; QUEIROZ, A. J. M.; OLIVEIRA, A. R. Características físico-químicas do fruto do mandacaru. In: Congresso Brasileiro de Ciência e Tecnologia de Alimentos, 19., 2004, Recife. Anais... Recife: SBCAT, 2004.

RUFINO, M. do S.M; ALVES, R. E.; BRITO, E. de S.B.; MORAIS, S.M. de ; SAMPAIO, C. de G.; PÉREZ-JIMÉNES,J.; SAURACALIXTO, F.D. Metodologia Científica: Determinação da Atividade Antioxidante Total em Frutas pela Captura do Radical Livre ABTS•+ .Comunicado Tecnico online, Julho, Fortaleza, 2007.

SILVA, A. C da. Potencial de resíduos agroindustriais como fontes de compostos bioativos. Tese (Doutorado em Ciência e Engenharia de Alimentos). Universidade Estadual Paulista Júlio Mesquista Filho. São Jose do Ribeirão Preto, 2014.

SILVA, A. O.; FILHO, A. M.; SILVA, J. A. S.; GORGONIO, C. R.; SILVA, C. M.; Caracterização físico - química da polpa e casca de frutos do mandacarú (cereus jamacaru). In: Encontro Nacional de Educação, Ciência e Tecnologia/UEPB. Campina grande, v.1, n.1, 2012.

SILVA, L. R.; ALVES, R. E.; Caracterização físico-química de frutos de mandacaru. Revista Acadêmica: Ciências Agrárias e Ambientais, Curitiba, v. 7, n.2, p. 199-205, abr/jun, 2009.

TAYLOR, N.; ZAPPI, D. Cacti of eastern Brazil. Kew:Royal Botanic Gardens, 2004. 
VIEIRA, L. M.; SOUSA, M. S. B.; MANCINI-FILHO, J.; LIMA, A. Fenólicos totais e capacidade antioxidante in vitro de polpas de frutos tropicais. Revista Brasileira de Fruticultura, Jaboticabal - SP, v. 33, n. 3, p. 888-897, 2011.

Waterhouse, A. Folin-ciocalteau micro method for total phenol in wine. American Journal of Enology and Viticulture, p. 3-5, 2006.

WINK, M. Secondary Metabolites, the Role in Plant Diversification. Academic press: Encyclopedia of Evolutionary Biology. Ed 11, 213p, 2016. 